\title{
Teaching Evaluation Algorithm Based on Grey Relational Analysis
}

\author{
Xiaoying Zhang, ${ }^{1}$ Xiuying Yang, ${ }^{1}$ and Jing Yang $\mathbb{D}^{2}$ \\ ${ }^{1}$ School of Finance, Hainan Vocational University of Science and Technology, Hainan 571126, China \\ ${ }^{2}$ Public Teaching Department, Hainan Vocational University of Science and Technology, Hainan 571126, China \\ Correspondence should be addressed to Jing Yang; yangjing@hvust.edu.cn
}

Received 30 January 2021; Revised 29 March 2021; Accepted 2 April 2021; Published 15 April 2021

Academic Editor: Wei Wang

Copyright (c) 2021 Xiaoying Zhang et al. This is an open access article distributed under the Creative Commons Attribution License, which permits unrestricted use, distribution, and reproduction in any medium, provided the original work is properly cited.

\begin{abstract}
Grey correlation analysis uses grey correlations to describe the strength, magnitude, and order of relationships among factors. The data sequence of the drmined data is used as a reference data string, and the data sequence matrix of each influence factor is used as a control data matrix to calculate the correlation between the data sequence and the reference of each factor in the control data matrix. The basic idea of quantitatively measuring the correlation between each factor and the object is to determine the similarity between the reference data sequence and the shape of multiple comparison data sequences and to determine whether the connection is strong. It reflects the degree of correlation between curves. In this paper, we examine the problem of evaluating the quality of undergraduate education by multiple indicators and establish a comprehensive evaluation model of the quality of undergraduate education in the 13 prefecture level cities by using the grey correlation analysis. The correlation coefficient of each index was obtained by the grey correlation analysis, the correlation number of each index was analyzed and ranked, and finally the improvement of the number of "advanced teachers" and the education of 13 prefectures were reduced as much as possible.
\end{abstract}

\section{Introduction}

Teaching is the basic way to achieve the purpose and task of education, and it is the most effective way to transfer knowledge and cultivate talents. Teaching directly affects students and is at the center of school education. The school's educational work must adhere to the principle of teaching as the main focus and carry out other work around teaching to promote the improvement of teaching quality [1]. In this period, teaching and life are the same thing, that is to say, when teaching and learning certain production and labor experience, social customs, and habits, there are sometimes inconsistencies. With the development of productive forces, the accumulation of experience and knowledge, and the emergence of schools, teaching was gradually separated from life itself, and teaching in its true sense emerged [2]. Around 20th century B.C., the words "teach" and "learn" already appeared in the Chinese oracle bones, and in the ancient text "Shang Shu-Saying Life," the phrase "teaching half" appeared. In the ancient Chinese "Shangshu Shushuosheng," the word "teaching" was first used in combination with the word "teaching". In ancient times, teaching and education were almost indistinguishable, and teaching was the whole activity in school. In the earliest educational document "Xueji," which discussed the theory of teaching, it was pointed out at the beginning that "teaching is the first thing to build the country and the people."

The "teaching" here is not the same as the "teaching" nowadays $[3,4]$. In the information world, there is not only a large amount of known information but also a lot of unknown information. The known information is white, the unknown information is black, and the space between white and black is gray. Grey system theory has three major types of systems: white, black, and grey. The white system is a system with sufficient and fully clear information [5-7]. For example, if you view Super Marquette or the store as a system, and the distribution of employees, capital flow, sales, and loss information is completely clear, then you can also determine the information about Super Marquette and the store, profit, and capital turnover [8]. And such systems can be classified as white systems. Black system is an entirely unknown system with 
insufficient information. For example, this planet system is a black system if the planet known to people is regarded as a system, but the volume, mass, and distance between planets and Earth are completely unknown [9]. The white system and the black system are relative, and in real life, these two cases are relatively rare [10]. Most systems have both clear and unclear information, between the white system and the black system, and such systems are called grey systems by Professor Deng Julong, as shown in Figure 1. With the development of teaching evaluation, a variety of evaluation methods have emerged, which can be generally summarized as qualitative evaluation, quantitative evaluation, and comprehensive evaluation methods combining qualitative and quantitative. Each evaluation method has its own characteristics and disadvantages. A specific evaluation process requires the appropriate evaluation method to be selected according to the actual needs of the evaluation object and evaluation. Grey correlation analysis methods study the grey system with incomplete and clear information. The maximum feature is that the structure is simple, the amount of calculation is small, and the number of samples is small, and it is not necessary to consider the distribution pattern of data at the time of analysis, so it is practicable. Therefore, in this paper, considering the ambiguity and uncertainty of factors affecting the quality of education, the grey correlation analysis method is applied to the evaluation of the quality of education of teachers. Correlation theory and evaluation results have strong objectivity and rationality.

In this paper, on the basis that the evaluation information is described in qualitative language, a teaching quality evaluation model based on cloud-grey correlation analysis is proposed for the grey characteristics exhibited by the teaching quality evaluation system, combined with the advantage that the cloud model can perform uncertainty conversion between qualitative concepts and quantitative values, and the feasibility of the model is analyzed and verified through specific examples. Focusing on the grey characteristics presented by the teacher teaching quality evaluation system and the unique advantages of grey correlation analysis in dealing with grey systems, the application of grey correlation analysis is proposed to evaluate teaching quality, and the basic steps and advantages of applying grey correlation analysis to determine index weights and evaluate teaching quality are introduced; in the solution of gray correlation degree, a series of discriminant coefficients are introduced to make up for the static value of insufficient discriminant coefficient; the method of evaluating teachers' teaching quality based on cloud-grey correlation analysis is proposed, which solves to a certain extent the difficult problem that evaluation indexes described by qualitative language are difficult to be accurately represented by mathematical models. The rest of the paper is organised as follows: Section 2 contains the literature review; Section 3 contains the methodology (method); Section 4 contains the results; and Section 5 contains the conclusions and policy implications.

\section{Related Work}

A grey system is a system in which the influencing factors are not completely clear and the relationship between factors is not completely clear. The concept of grey system evolved from the "black box" theory proposed by the British scientist William Ross Ashby, which describes some internal structure, parameters, characteristics, and other information that are completely unknown and can only be studied through external input-output relationships. The "black box" describes a class of things whose internal structure, parameters, and properties are completely unknown and can only be studied through external input-output relationships. Grey system theory, on the other hand, advocates research based on the internal information of the system, eliminating some of the drawbacks of the "black box" theory, so as to conduct system research more accurately. With the continuous exploration of grey system theory, scholars in the United States, Germany, the United Kingdom, the Netherlands, Australia, and other countries, as well as Hong Kong, Macao, and Taiwan, have conducted a lot of research on its application in different fields such as military, meteorology, medicine, engineering, and agriculture and have achieved a series of research results. For example, in the field of agriculture, the former applied grey system theory to the dynamic analysis and trend prediction of grain production in Hubei Province, which provided a theoretical basis for the development and decision making of grain production; in the field of medicine, researchers applied grey system theory to the diagnosis of hepatitis and cirrhosis in Chinese medicine and proposed a new diagnosis method based on grey correlation analysis, which promoted the development of modernization and objectivity in Chinese medicine [11].

Many scholars at home and abroad have devoted themselves to the research of grey system theory and have published papers on grey system theory in more than 200 international and domestic academic journals, and more than 200 grey system research results have won national or provincial awards, and many important international conferences have included grey system theory as a discussion topic for discussion [12]. For example, the IEEE International Conference on Systems, Man, and Control has arranged sessions on grey systems for five consecutive years since 2004 in the Hague, Netherlands; Arizona, USA; Mariupol, Slovenia; Hawaii, USA; Taiwan, China; Montreal, Canada; Wroclaw, Poland; and Singapore [13]. In 2003, the 32nd International Conference on Computer and Industrial Engineering in Ireland arranged four sessions on grey system theory $[14,15]$; in addition, the International Conference on System Predictive Control and the International Conference on Modeling of Uncertainty Systems have made grey system theory a hot topic of their attention and discussion [16-18].

\section{Evaluation Index System of Grey Correlation Analysis}

An indicator is a specific behavioral evaluation criterion and a unit of measurement target. The indicator system is a systematic group of indicators composed of several indicators in a certain order and structure, which are decomposed according to certain objectives to decompose the attributes of the evaluation object one by one. In the process 

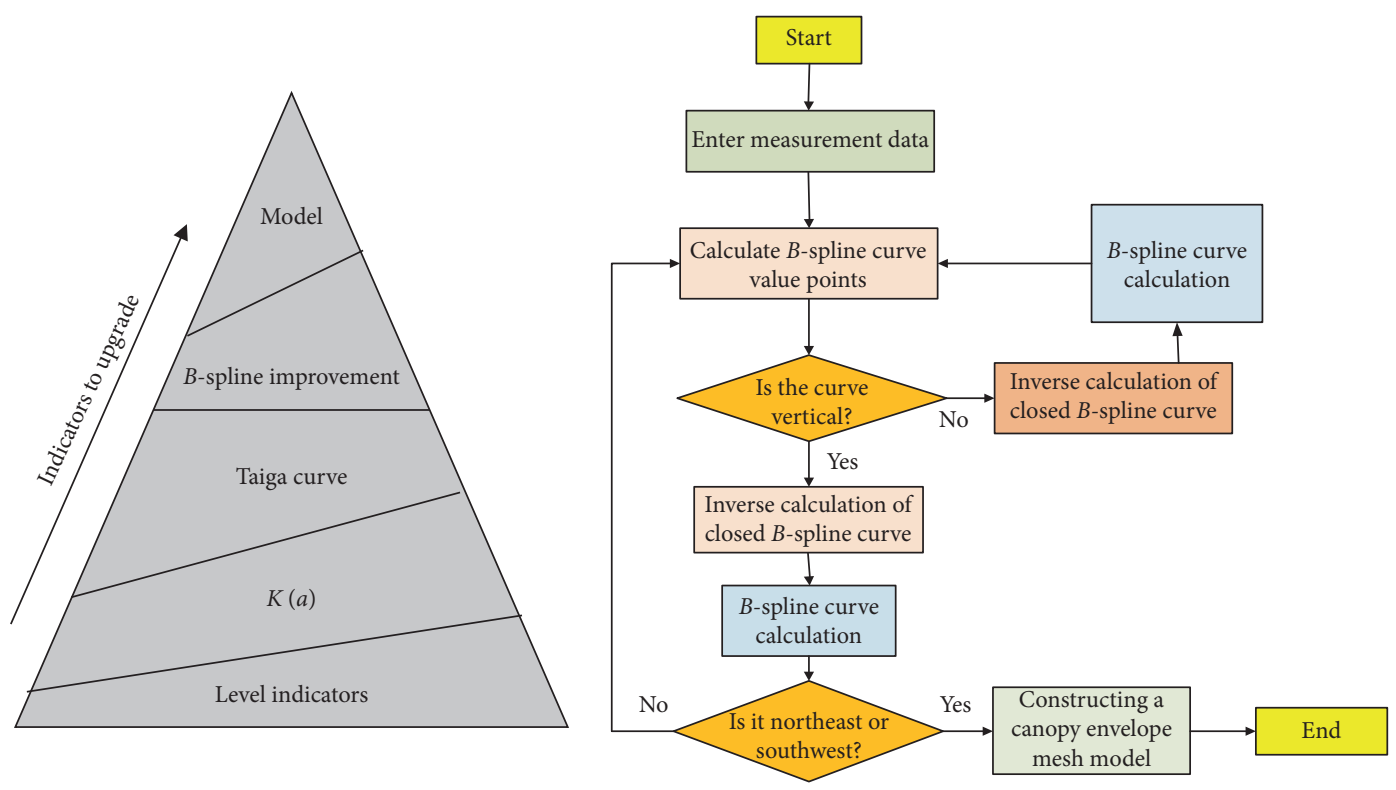

FiguRE 1: Tower structure of greyscale evaluation index system.

of teaching quality evaluation, the evaluation index system is the basic basis for teaching evaluation, and whether the evaluation index system is scientific and reasonable directly affects the reasonableness and effectiveness of the teaching quality evaluation results. Therefore, the establishment of a scientific and teaching quality evaluation system associated with teaching activities is crucial in the whole teaching quality evaluation activity.

In the process of teaching quality evaluation, the factors affecting teaching quality have complex correlations and are of certain complexity; therefore, in the process of designing the evaluation index system, the relevant principles of evaluation index system construction should be followed so as to build a reasonable and effective evaluation index system structure.

3.1. Scientific Principle. The principle of scientificity refers to the fact that in the process of teaching evaluation, its evaluation index system should have a certain theoretical basis, and all the indexes should be consistent with the intended objectives. Secondly, the concept description of each index should be scientific and accurate, the scope of calculation should be clear, and the indexes closely related to teaching quality should be analyzed and selected comprehensively so that the index system can reflect the essential characteristics of the evaluation object as reasonably and effectively as possible.

3.2. Feasibility Principle. The principle of feasibility means that in the process of designing the teaching evaluation index system, the evaluation indexes should be simple and clear, the content of the indexes should have clear connotations and be measurable, and the data of the indexes should be easy to obtain and simple to handle, so as to ensure that the whole evaluation can be carried out smoothly.
3.3. Comparability Principle. The principle of comparability is to require that the evaluation indexes must be the common attributes of all evaluation objects, reflecting the qualitative consistency. In addition, since the quantity of different things can be compared with each other quantitatively only after they are transformed into the same unit, the meaning, scope, and unit of measurement of evaluation indexes must be consistent so that they are comparable, and the stronger their comparability is, the more credible the final evaluation results will be.

3.4. Principle of Accuracy. The principle of accuracy means that when selecting evaluation indexes, the objective laws of teaching activities should be followed, combined with the objective reality of teaching activities, reflecting the essence of teaching work, the selected evaluation indexes should have precise connotation and extension and should accurately reflect the actual teaching profile of teachers, and the established index system should be objective and credible and should accurately reflect the true level of teaching evaluation.

3.5. Independence Principle. The principle of independence means that the indicators in the teaching quality evaluation index system should maintain a certain relative independence, not overlapping and not subordinate to each other, and the indicators at the same level can only be in juxtaposition with each other, and there cannot be the relationship of inclusion and inclusion or cause-effect relationship. The reason is if the indicators in the index system are not independent of each other, then there will be redundant indicators, which will increase the workload of the whole evaluation process and lead to the reduction of the feasibility of the evaluation results; in addition, if the indicators are included in each other, then in the specific 
evaluation process, the indicators will be scored several times, which will increase their corresponding weights and affect the final evaluation results.

\subsubsection{Basic Steps of Grey Correlation Analysis Algorithm.} The specific calculation steps of the grey correlation analysis are as follows.

Step 1. Determine the analysis series.

Determine the reference series that reflects the characteristics of the system behavior and the comparison series that affects the system behavior. The data series reflecting the characteristics of the system behavior is called the reference series. The data series consisting of factors that influence the system behavior is called the comparison series. be

Let the reference series (also known as the parent series)

$$
Q=\left\{Q_{a}(i) \mid i=1,2, \ldots, n\right\}
$$

The comparison series (also known as subsequence) are

$$
P_{k}=\left\{P_{a}(i) \mid i=1,2, \ldots, n\right\}, k=1,2, \ldots, n .
$$

Step 2. The variables are dimensionless.

Since the data in each factor column in the system may differ in magnitude, it is not easy to compare or it is difficult to get the correct conclusion when comparing. Therefore, dimensionless processing of data is generally performed when performing grey correlation analysis.

Step 3. Calculate the number of correlation coefficients.

The number of correlation coefficients between $x_{0}(k)$ and $x_{i}(k)$ :

$$
\chi_{a}(k)=\frac{\min \max k(a)-y_{a}(i)}{\beta \rho \max k(a)} .
$$

Letting $\Delta_{a}(k)=k(a)-y_{a}(i)$, we have

$$
\chi_{a}(k)=\frac{\min \max \Delta_{a}(k)}{\beta \rho \max k(a)} .
$$

The smaller $\rho$, the greater the resolution, and generally the value of $\rho$ is $(0,1)$, depending on the situation. When $\rho \leq 0.5463$, the best discriminative power is obtained, and $\rho=0.5$ is usually taken.

Step 4. Calculate the correlation degree.

Because the correlation coefficient is the value of the degree of correlation between the comparison series and the reference series at each moment (i.e., each point in the curve), it has more than one number, and the information is too scattered to facilitate a holistic comparison. Therefore, it is necessary to concentrate the correlation coefficients of each moment (i.e., each point in the curve) into a single value, i.e., its average value, as a quantitative representation of the degree of correlation between the comparison series and the reference series.
Step 5. Relevance sorting

The correlations are ordered by size, and if $r_{1}<r_{2}$, then the reference series $y$ is more similar to the comparison series $x_{2}$. After calculating the correlation coefficients of the $X_{i}(k)$ series with the $Y(k)$ series, the average of the various types of correlation coefficients is calculated, and the average $r_{i}$ is called the correlation degree of $Y(k)$ with $X_{i}(k)$.

3.5.2. The Overall Architecture and Functional Composition of the System Design. The teaching evaluation system can have a separate data server or share the data server with the existing academic affairs management system. The administrator manages and publishes various information of teaching evaluation through Internet and divides the public level of information according to the situation; the evaluation subjects (students, teachers' peers, and experts) also submit evaluation information and browse the statistical result reports made public to themselves through Internet. In this paper, the weight value of each evaluation index is firstly determined by the grey correlation analysis algorithm. The comparative judgment matrix of the criterion layer and each element in each criterion layer is firstly constructed and then quantified by using the scale, and the relative importance of each element is calculated, as shown in Figure 2.

$\lambda_{\max }=5.032, C_{I}=0.046$, and $R_{I}=1.210 . \quad C_{R}=C_{I} /$ $R_{I}=0.034<0.1$ satisfied the requirement of consistency. Due to the limitation of space, the weights of the indicators at each level are not given in this paper. Assuming that the teaching management of five departments, A, B, C, D, and E, is reasonably evaluated, in order to conduct an objective and fair evaluation, six experts are asked to evaluate and score 20 indicators of each faculty, and the interval of equal scores is set to a minimum of 1 and a maximum of 10; all these indicators are scored as large as possible, and the structure of the scores given by the experts is quantified by the whitening weight function, and this paper takes $C 3$ teaching program evaluation as an example for illustration; this paper makes $u=u_{\max }=9$. Original Whitening value is $v=1$, when $u=u_{\min }=5$, whitening value is $v=0$; this paper establishes the whitening weight function as

$$
v=\frac{1}{6} q-1 .
$$

The corresponding whitening values $v_{i},\left\{v_{i}\right\}=\{0.643$, $0.459,0.783,0.400,0.574\}$ can be obtained from the whitening weight function. By the same token, the whitening values of the other indicators can be calculated. Next, the grey correlation coefficient $\xi_{i}(k)$ needs to be found, by means of the following equations:

$$
\begin{aligned}
\Delta_{a}(q) & =\left\{k=1 \mid x_{o}(k)-x_{i}(k)\right\}, \\
\lambda_{a}(i) & =\frac{\max \min \Delta_{a}(q)}{\Delta_{a}(q)+\delta \max \left(\Delta i(q) / \min \Delta_{a}(q)\right) .}
\end{aligned}
$$

Combine the correlation coefficients as well as the weights, by weighting the grey correlations:

$$
q_{a}=\frac{\sum_{i=1}^{n} v_{i} \lambda_{i}}{n}
$$




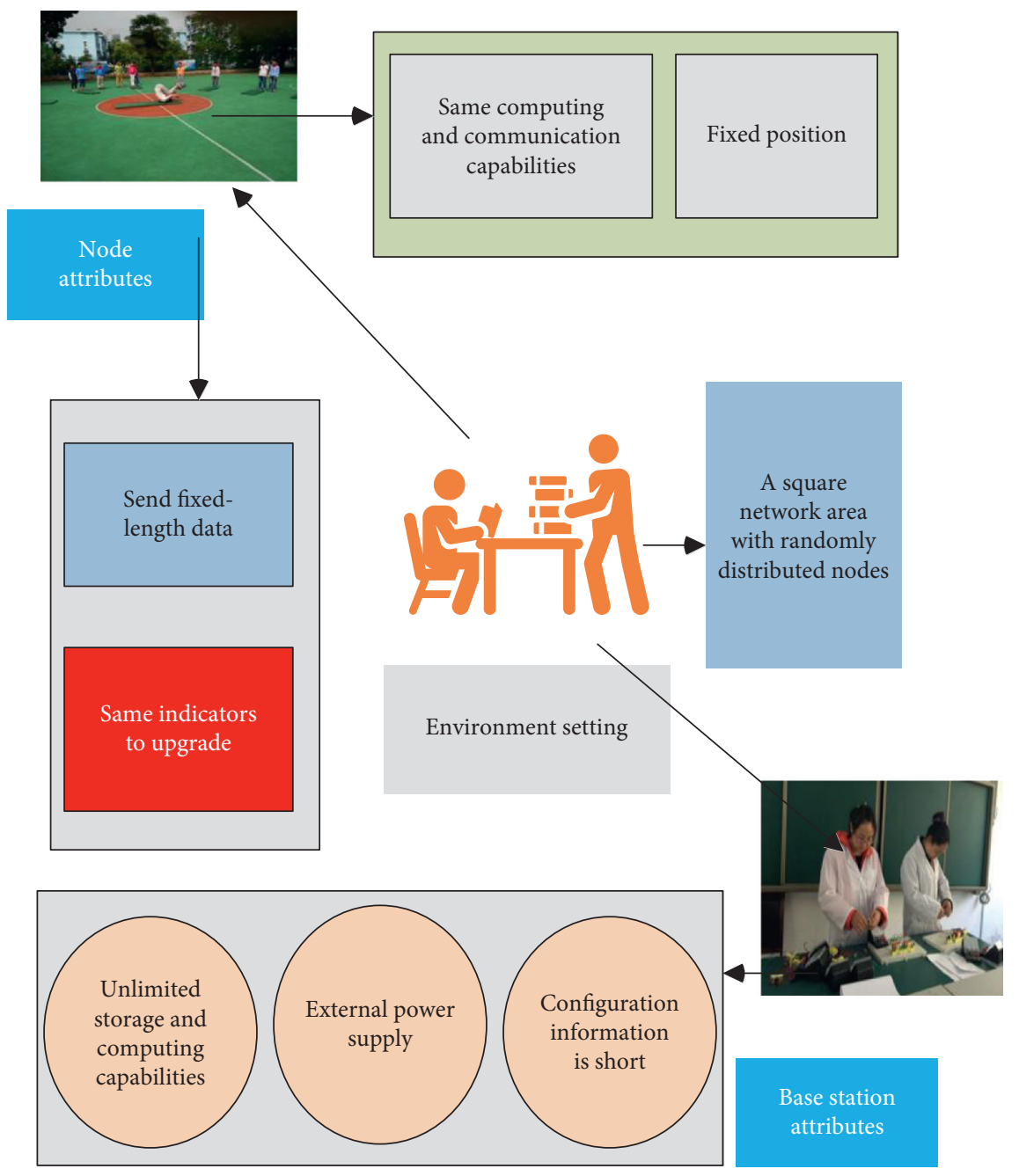

FIgURE 2: Weighting of indicators at each level.

We calculate the relevance of the teaching management of each department and compare the scale rankings according to the gray correlation ri. The example results show that through the analysis of various indicators, the department $\mathrm{D}$ with the most is the best.

\section{Comprehensive Evaluation of Teaching Quality Based on Grey Correlation Analysis}

4.1. Calculation of Individual Employment Quality Evaluation Coefficients. The individual employment quality evaluation index is mainly for individual students' evaluation. The employment quality evaluation value of individual students is obtained by analyzing the individual employment data of a certain sample size of students in the university. The calculation of individual employment quality evaluation value is done by normalizing the initial data of 14 index factors and then conducting grey correlation analysis on the normalized data to get the correlation degree between factors and calculating the weight value according to the correlation degree. The personal employment quality evaluation value not only reflects the employment situation of college graduates but also is a key indicator in the employment quality evaluation model. The higher the individual employment evaluation is, the more it can visually illustrate the excellent employment quality of the graduates. Based on the analysis of the graduate employment data of a quantitative sample of a university, the individual employment evaluation value of some graduates in the sample is obtained. Firstly, 1573 sample data are quantified to obtain the data in Figure 3, then the quantified data are normalized to obtain the partial sample data, and then the normalized data are analyzed and processed by using the constructed employment quality evaluation model, and the grey correlation analysis model is established separately to calculate the correlation degree between each indicator series and the employment quality of the reference series, and the value of the correlation degree is used to determine the weight of each indicator by using the hierarchical analysis model to determine the weight of each indicator and calculate the evaluation coefficient of individual employment quality according to the formula of the rating value and weight of the indicator. 


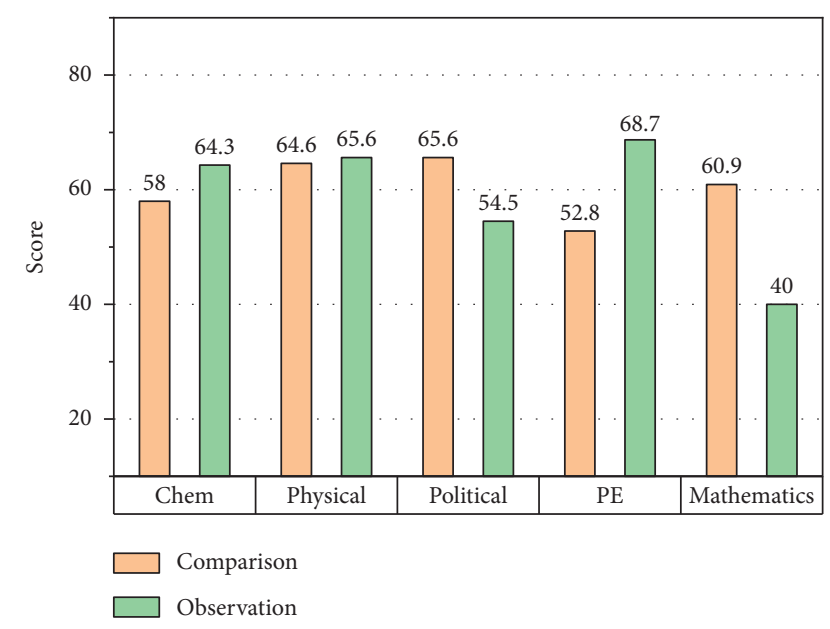

FIgUre 3: Quantified partial data.

A1-A14 indicate the degree of job and major relevance, salary income, availability of five insurance and one fund, nature of work unit, overall satisfaction with innovative education of home university, whether they received employment assistance, degree of social demand of their majors, overall satisfaction with home university, job adaptation period, job salary satisfaction, job development prospect satisfaction, satisfaction with the effect of employment consultation and guidance, satisfaction with employment assistance and measures, and degree of social demand of their majors. Each of the 14 indicators is quantified by numbers, which is convenient for the researcher to analyze the employment data of graduates. Figure 3 reflects the correlation values of each indicator. The highest correlation between each indicator and the employment quality of the reference series was 0.791 , the lowest correlation was 0.468 , and the correlations of other indicators were maintained between 0.5 and 0.8 .

4.2. Calculation of Indicator Weights. According to the correlation degree of individual indexes obtained from the above figure, the difference matrix is constructed by applying the steps of determining weights in the established evaluation model of employment quality of college graduates, and finally, the obtained data are shown in Figure 4 (three decimal places are retained).

According to the characteristics of the judgment matrix, $a_{i j}>0$ and $a a_{j i i j}>1$; when the difference between two indicators is negative, the reciprocal of its absolute value is taken as the sequence value; according to the scaling method of the judgment matrix, when the difference between two indicators is between 0.1 and 0.2 , its scale is 1 , which means that the two indicators are equally important. When the difference between two indicators is below 0.1 , the scale is 1 , which means that the two indicators are equally important, and when the difference between two indicators is between 0.1 and 0.2 , the scale is 2 , such as $a_{2,3}=0.125$, whose value is between 0.1 and 0.2 , so the scale is 2 . -0.1112 , whose absolute value is between 0.1 and 0.2 , so its scale is $1 / 2$, and so on, and finally the judgment matrix is constructed. The judgment
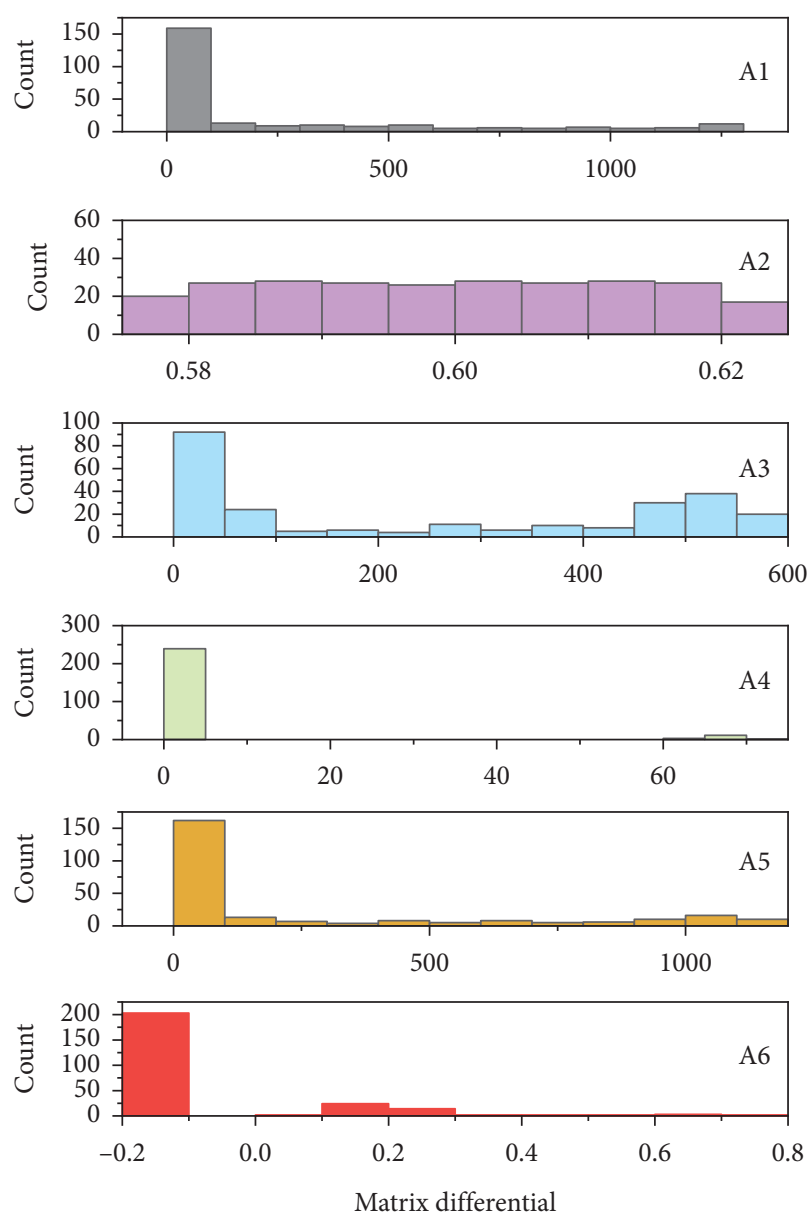

Figure 4: Difference matrix data.

matrix is shown in Figure 5. A1-A14 correspond to 14 indicators; the diagonal values of the matrix are 1, and $a i i=1$; the values of the two sides of the matrix diagonal are inverse of each other. According to the nature of the judgment matrix, Matlab software is used to run the procedure of hierarchical analysis model, and the weights of each indicator are obtained by the formula, and the consistency test is conducted according to the average random consistency index list and the indicators, in which the eigenvalue is 14.394 , the consistency ratio CR is 0.0192 , which is less than 0.1 , and the consistency index CI is 0.030 , which is in the range of the average.

\section{Results and Discussion}

Professional employment evaluation is mainly the evaluation of professional employment quality obtained by different majors in individual employment data. The professional employment quality situation not only reflects the professional setting of colleges and universities but also is a credential that reflects the relevance of colleges and universities to social development. According to the analysis of employment data of graduates from a university, we know that the university has set up the following majors: material forming and control 


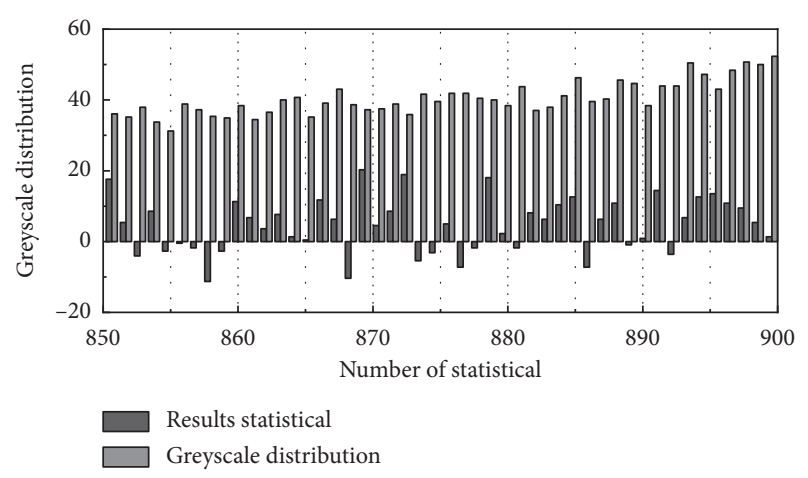

FIgURE 5: The judgment matrix by the formula.

engineering, measurement and control technology and instrument, urban and rural planning, road, bridge, and river crossing engineering, geographic information science, electronic information science and technology, port, waterway, and coastal engineering, industrial engineering, international economics and trade, environmental design, architecture, transportation, labor and social security, marketing, and visual communication design.

There are 59 majors, such as Inorganic Nonmetallic Materials Engineering, Logistics Management, Information Management and Information System, and Applied Statistics. Professional employment evaluation index further reflects the employment evaluation of personal employment data, and personal employment evaluation is the premise of professional employment evaluation. The higher the professional employment evaluation value is, the higher the employment quality of the profession is, and thus the better the relevance of the profession in the university and the society. According to the employment data applied to the employment quality evaluation model for inductive analysis, the mean value of individual employment quality evaluation of different majors is obtained. The values of professional employment evaluation coefficients are calculated according to the formula, and the data are shown in Figure 6.

Geography and Information Science, Urban and Underground Engineering, Computer Science and Technology, and Engineering Management are the top four majors in terms of evaluation value among all majors, all of which exceed 3.3. The employment evaluation value of Geography and Information Science is the highest at 3.397, followed by Urban and Underground Engineering at 3.382, Computer Science and Technology at 3.376, and Engineering Management at 3.372. Among these fifty-nine majors, the evaluation coefficients of four majors, namely, Russian, Industrial Engineering, Resource Recycling Science and Engineering, and Chinese International Education, do not exceed 3.0, while most of the others exceed 3.0 and remain stable between 3.0 and 3.3 .

All of the remaining four major coefficients are over 3.0 and are stable between 3.0 and 3.3. It can be seen that the quality of employment of each major university is more valuable. All major universities are classified into art and science. The majors of liberal arts include philosophy,

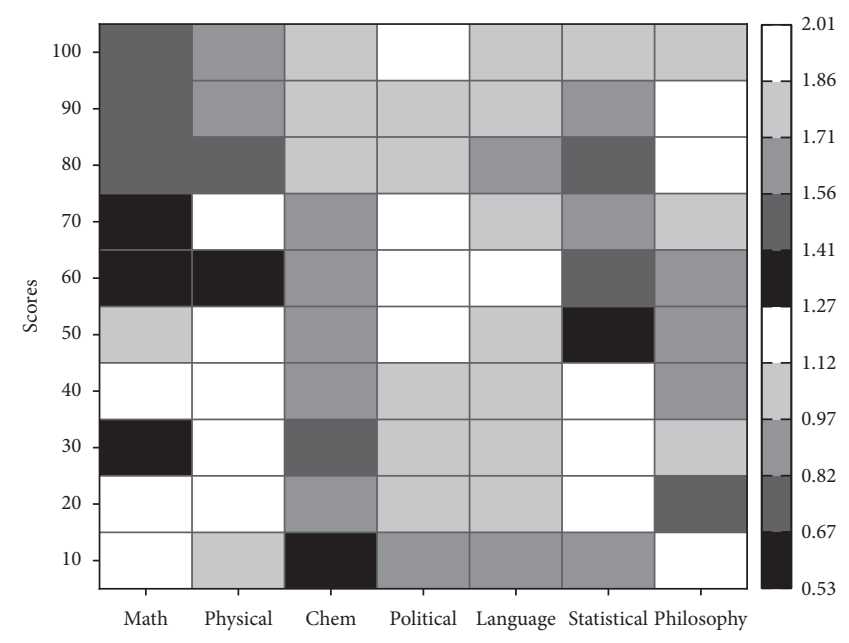

Figure 6: Professional employment evaluation index values.

economics, law, education, literature, history, and so on; the majors of science are mainly theoretical studies, including mathematics, physics, chemistry, biological science, astronomy, atmospheric science, and electronic information science; the majors of engineering are mainly applicationoriented, cultivating technology and techniques (or engineering and technology), including civil engineering, highway and bridge, machinery, water conservancy, and electrical. The disciplines covered include civil engineering, highway and bridge, mechanical, hydraulic, electrical, automotive, electronics and information, thermal and nuclear energy, materials, instrumentation, environmental engineering, pharmacy and pharmaceutical, aerospace, etc. Through the statistical analysis of the collected data, the employment quality evaluation coefficient of the category is calculated according to the formula, and the evaluation value is obtained by dividing the number of majors by the sum of the employment quality evaluation coefficients of the majors included in the category. The obtained division of majors in arts and sciences is shown in Figure 7.

The application of personal employment evaluation index, professional employment evaluation index, and section employment evaluation index in the employment quality evaluation model illustrates that the employment quality of the university is generally good, as shown by the personal employment evaluation value, professional employment evaluation value, and section employment evaluation value. In the individual employment evaluation value, more than half of the graduates' employment quality evaluation value exceeds the average value. Among the professional employment evaluation coefficients, the employment evaluation coefficient of geographic information science is the highest, the second ranking is urban underground space engineering, and the third is computer science and technology; among the subject category employment evaluation coefficients, the employment evaluation coefficient of engineering is the highest, and the employment evaluation coefficient of liberal arts is the lowest, which is consistent with the university's professional. 

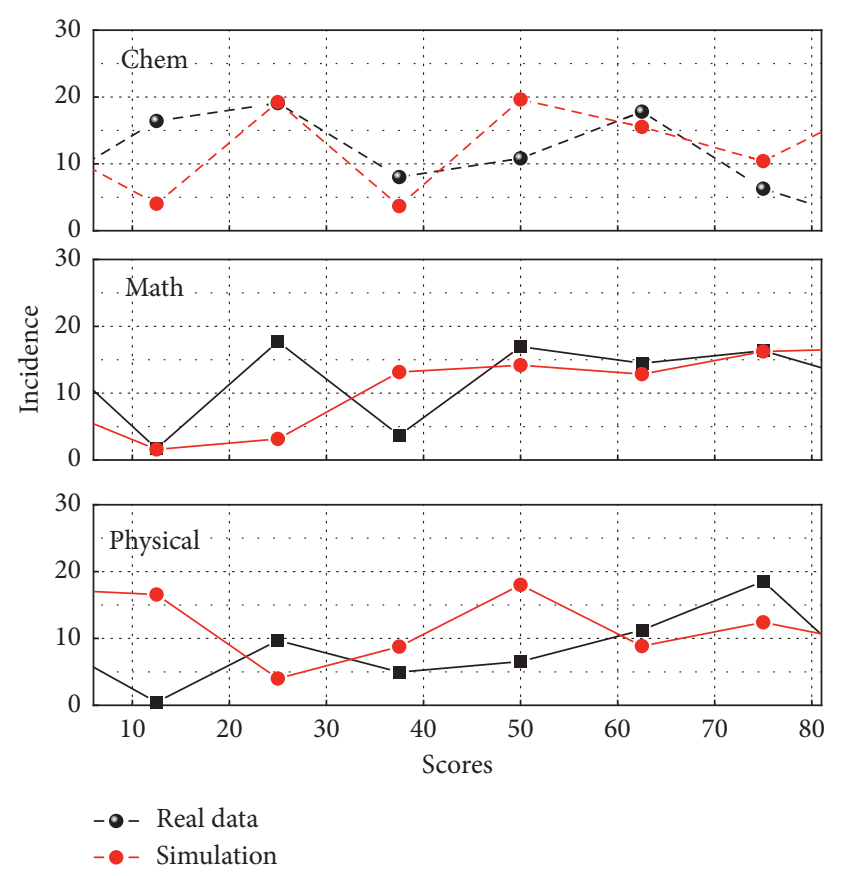

Figure 7: Classification of academic disciplines.

\section{Conclusion}

Improving the quality of education is always a theme in the process of education and education, and the scientific and rational evaluation of the quality of teachers' education is an important means of maintaining the steady development of the education system. This paper shows the basic theories and general methods for teaching teachers to evaluate quality through the research of relevant literature. We focus on the proprietary advantages of grey characteristics presented by the teacher's quality evaluation system and the greyscale analysis of grey correlation analysis in dealing with grey systems. We introduce basic procedures and advantages of grey correlation analysis to determine the weight of the index and evaluate the quality of education. In the solution of grey correlation, a series of discriminant coefficients is introduced to compensate for the defect of static acquisition of the discriminant coefficient. A method for evaluating the quality of education of teachers based on cloud correlation analysis has been proposed. A method of evaluating the quality of education of teachers based on grey correlation analysis is built in MATLAB. A teacher evaluation model of teacher education based on grey correlation analysis was constructed in MATLAB. The final analysis shows that the teacher's teaching quality evaluation method based on the grey correlation analysis is reasonable and is a viable new method for teaching evaluation.

\section{Data Availability}

The data used to support the findings of this study are available from the corresponding author upon request.

\section{Conflicts of Interest}

The authors declare that they have no conflicts of interest.

\section{Acknowledgments}

This research was supported by the Education and Teaching Reform Project of Higher Education in Hainan Province "Research on the Influence of Course Teaching Method on Students' Learning Motivation and Learning Effect" (Hnkj2019-134).

\section{References}

[1] V. Albert, S. Mndolo, E. M. Harrison, E. O’Sullivan, I. H. Wilson, and I. A. Walker, "Lifebox pulse oximeter implementation in Malawi: evaluation of educational outcomes and impact on oxygen desaturation episodes during anaesthesia," Anaesthesia, vol. 72, no. 6, pp. 686-693, 2017.

[2] S. O. N. Raj and S. Prabhu, "Modeling and analysis of titanium alloy in wire-cut edm using grey relation coupled with principle component analysis," Australian Journal of Mechanical Engineering, vol. 15, no. 3, pp. 198-209, 2017.

[3] Q. Lin, Y. Zhu, S. Zhang, P. Shi, Q. Guo, and Z. Niu, "Lexical based automated teaching evaluation via students' short reviews," Computer Applications in Engineering Education, vol. 27, no. 1, pp. 194-205, 2019.

[4] C.-C. Hsu, J. J. H. Liou, and Y.-C. Chuang, "Integrating DANP and modified grey relation theory for the selection of an outsourcing provider," Expert Systems with Applications, vol. 40, no. 6, pp. 2297-2304, 2013.

[5] L. Myerholtz, A. Reid, H. M. Baker, L. Rollins, and C. P. Page, "Residency faculty teaching evaluation: what do faculty, residents, and program directors want?" Family Medicine, vol. 51, no. 6, pp. 509-515, 2019.

[6] Y. Wang, Y. Huang, X. Zeng et al., "Faulty feeder detection of single phase-earth fault using grey relation degree in resonant grounding system," Ieee Transactions on Power Delivery, vol. 32, no. 1, pp. 55-61, 2017.

[7] N. Huang, "Analysis and design of university teaching evaluation system based on JSP platform," International Journal of Education and Management Engineering, vol. 7, no. 3, pp. 43-50, 2017.

[8] Q. Wang, C. Wu, and Y. Sun, "Evaluating corporate social responsibility of airlines using entropy weight and grey relation analysis," Journal of Air Transport Management, vol. 42, pp. 55-62, 2015.

[9] T. S. Riall, J. Teiman, M. Chang et al., "Maintaining the fire but avoiding burnout: implementation and evaluation of a resident well-being program," Journal of the American College of Surgeons, vol. 226, no. 4, pp. 369-379, 2017.

[10] T. Singh, A. Patnaik, and R. Chauhan, "Optimization of tribological properties of cement kiln dust-filled brake pad using grey relation analysis," Materials \& Design, vol. 89, pp. 1335-1342, 2016.

[11] M.-F. Chen and G.-H. Tzeng, "Combining grey relation and topsis concepts for selecting an expatriate host country," Mathematical and Computer Modelling, vol. 40, no. 13, pp. 1473-1490, 2004.

[12] M.-F. Chen, "Decision-making method based on grey relation analysis and trapezoidal fuzzy neutrosophic numbers under double incomplete information and its application in typhoon disaster assessment," Materials \& Design, vol. 4, pp. 36063628, 2019. 
[13] T. Wang, "Study on adhesion property of asphalt and aggregate based on grey relation," Theory, vol. 48, no. 14, pp. 40-42, 2019.

[14] J. W. Boland, M. E. L. Brown, A. Duenas, G. M. Finn, and J. J. B. O. Gibbins, "How effective is undergraduate palliative care teaching for medical students?" A Systematic Literature Review, vol. 10, no. 9, 2020.

[15] J. J. J. O U. Teaching and L. Practice, "A practice-based study of Chinese students," Learning-Putting Things Together, vol. 16, no. 2, pp. 12-18, 2019.

[16] X. J. J. O P. C. Wang, "Application of grey relation analysis theory to choose high reliability of the network," Node, vol. 1237, no. 3, 2019.

[17] W. Wang, X. Zhao, Z. Gong, Z. Chen, N. Zhang, and W. Wei, "An attention-based deep learning framework for trip destination prediction of sharing bike," IEEE Transactions on Intelligent Transportation Systems, vol. 1, 2020.

[18] S. Xia, D. Peng, D. Meng et al., "A fast adaptive k-means with No bounds," IEEE Transactions on Pattern Analysis and Machine Intelligence, vol. 1, 2020. 